\title{
A study of phase-steepened Alfvén waves in a high-speed stream at $0.29 \mathrm{AU}$
}

\author{
P. Alexander \\ Departamento de Física, Facultad de Ciencias Exactas y Naturales, Universidad de Buenos Aires, Argentina \\ e-mail: peter@df.uba.ar
}

Received: 20 September 1999 / Revised: 27 March 2000 / Accepted: 14 April 2000

\begin{abstract}
This work performs a search of phase-steepened Alfvén waves under a priori ideal conditions: a high-speed solar wind stream observed in one of the closest approaches to the Sun by any spacecraft (Helios 2). Five potential candidates were initially found following procedures established in earlier work. The observed cases exhibited arc-like or elliptical polarizations, and the rotational discontinuities that formed the abrupt wave edges were found at either the leading or the trailing part. The consideration of some additional specific parameters (mainly related to the relative orientation between mean magnetic field, wave and discontinuity) has been suggested here for an ultimate and proper identification of this kind of phenomenon. After the inclusion of these calculations in our analysis, even fewer cases than the five originals remain. It is suggested that optimum conditions for the detection rather than just for the existence of these events have to be reconsidered.
\end{abstract}

Key words: Interplanetary physics (discontinuities; MHD waves and turbulence; solar wind plasma)

\section{Introduction}

The study of fluctuations in the solar wind divided, almost from the beginning, into two diverse basic viewpoints: Coleman (1968) proposed that the fluctuations are of a turbulent nature while Belcher and Davis (1971) suggested that they are due to propagating Alfvén waves. Later, Tu and Marsch (1992) presented a model aimed at reconciling these two interpretations. They assume that the solar wind fluctuations are composed of both turbulent convective structures and Alfvén waves propagating both inwards and outwards along magnetic field lines. A significant dominance of outward Alfvén modes has been found in fast streams near the Sun (see e.g. Marsch, 1991).
Early investigations of the interplanetary magnetic field (IMF) revealed occasional changes in its direction that are abrupt on a time scale of seconds. These features were termed directional discontinuities (Burlaga, 1969). They were later discriminated into tangential or rotational according to their characteristics: the former possess no field normal to the discontinuity surface but show a significant change in the magnitude and direction of the tangential component crossing it and they represent nonpragating borders between two different plasma regimes, whereas the latter exhibit a constant magnetic field component normal to the surface, for thermally isotropic plasmas the magnitude of their tangential field does not change, and they image sharply crested Alfvén waves (sometimes referred to as Alfvén shocks). However, these descriptions apply to theoretical cases, but the solar wind plasma does generally not behave very much like that. Discontinuities have been rarely observed with ideal properties (within measurement errors) and about one or two are usually detected per hour by a spaceprobe at $1 \mathrm{AU}$.

While analyzing a possible relationship between solar wind bulk structure and discontinuities, Turner (1973) noticed that rotational cases appear preferentially embedded in Alfvénic fluctuations. Barnstorf (1980) mentioned the need for a closer inspection of the link apparent in a persistent joint appearance of these phenomena and remarked that in high-speed streams the occurrence rate of directional discontinuities is largest. The number of interplanetary discontinuities has been definitely found to be high in stream-stream interaction regions and in Alfvén wave trains, which in particular dominate high-speed streams, but in this last case it is due to the significant presence of outward propagating waves rather than to the large convection speed (Tsurutani et al., 1994). Moreover, discontinuities occurred at the edges of the more slowly rotating Alfvén waves in about $50 \%$ of the examined cases of Ulysses data (Tsurutani et al., 1994, 1997a). Then, it seems probable that many discontinuities, especially those present in high-speed streams, are of a rotational nature, 
and furthermore, they could be an integral part of an Alfvén wave. The slowly turning part and the fast portion respectively comprise a considerable fraction of one rotation of the magnetic field, and they both complete together a $360^{\circ}$ gyration in the same plane. It appears as though the rotational discontinuity is the phase-steepened edge of an Alfvén wave, as the phase rotation rate is not uniform but speeds up at one of the extremes.

A sound knowledge of the behaviour of the IMF in different circumstances and conditions is important for many areas of heliospheric research. In particular, magnetohydrodynamic nonlinearities have been extensively studied in the solar wind during the last decade (see e.g. Tu and Marsch, 1995 and references therein). We will focus here on the detection of the mentioned interplanetary phase-steepened Alfvén waves. Optimum conditions for their occurrence should be found in highspeed streams at short heliocentric distances. This work deals with one out of some of the closest approaches of a spaceprobe to the Sun to date, and which particularly found a persistent flow of fast solar wind.

\section{Data analysis}

The Helios mission was aimed at performing in situ measurements of the interplanetary medium in the nearsolar environment. Two almost identical spacecraft were launched on December 1974 and January 1976 into highly elliptical, small inclination orbits with a perihelion distance of $0.31 \mathrm{AU}$ for Helios 1 and $0.29 \mathrm{AU}$ for Helios 2. The perihelion of the latter rather than the former has been chosen for the present study, because of a better coverage of the combined plasma and IMF data. This spacecraft made eight approaches to the Sun. It is quite fortunate that the interval with the best highresolution data coincides with an excellent period from the Alfvénic point of view: an immersion in a high-speed stream with no magnetic polarity reversal. This may be seen in Fig. 1, which shows the solar wind speed and magnetic field longitude between days 105-110 1976 (the spacecraft stays at 0.29 AU between 9 PM of day 105 and $8 \mathrm{AM}$ of day 110). The IMF vectorial data consist of the three components according to the HSE system, i.e. $x$ points from Helios spacecraft to the Sun, $z$ points to the north pole and $y$ completes a right-hand system. There are typically four magnetic field vector measurements every second and the plasma parameters are sampled every $40.5 \mathrm{~s}$. The IMF angle represented in Fig. 1 corresponds to interpolations in the same intervals. By calculating the correlation coefficients at zero lag between the fluctuations in solar wind velocity and in magnetic field, for days 105-110, we find an average of $-0.8,-0.9$ and -0.9 in the $x, y, z$ directions. This is consistent with the region being dominated by Alfvénic fluctuations propagating radially outward.

An automation of the identification of interplanetary directional discontinuities by computer has several advantages: elimination of human error and bias and the possibility of processing large amounts of data.

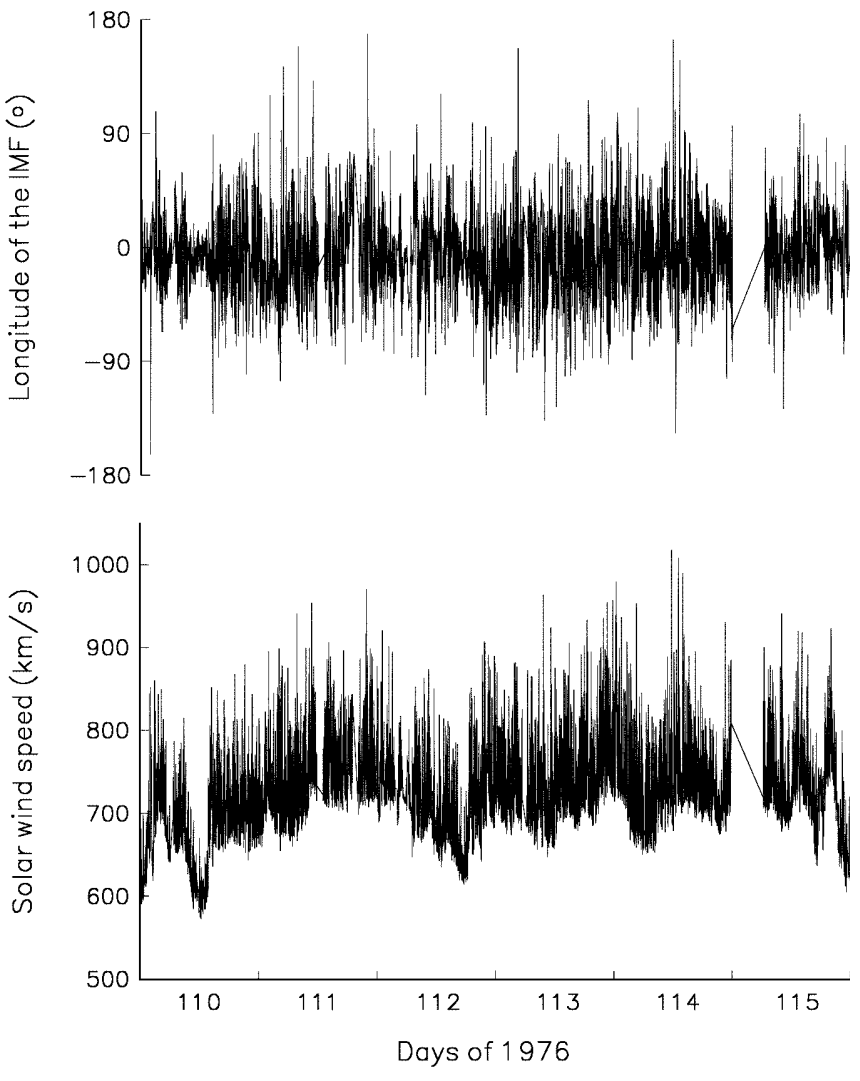

Fig. 1. Helios 2 observations of solar wind speed and IMF longitude $\left(0^{\circ}\right.$ means away from the Sun, positive angles are measured anticlockwise) during the days analyzed

Many criteria have been developed (see e.g. Tsurutani and Smith, 1979; Lepping and Behannon, 1986) and the differences among them are given by the requirements and constraints for the appropriate occurrence of a sharp change in the direction of the magnetic field. Burlaga (1968) required that there be variations in field direction greater than $30^{\circ}$ in less than $30 \mathrm{~s}$ and later works usually followed approximately this definition (see e.g. Burlaga, 1995). Additional conditions that allow one to discard similar phenomena that might be wrongly included depend on the heliocentric zone for which each criteria set has been designed, because a thickening of discontinuities (Tsurutani and Smith, 1979; Lepping and Behannon, 1986) and a decrease of field strength occurs for increasing heliocentric distance.

Our identification process was adopted from Barnstorf (1980) because it was particularly set up for the region visited by Helios spacecraft $(0.3-1.0 \mathrm{AU})$. The jump condition we applied to our $40.5 \mathrm{~s}$ set of magnetic field vectors $\mathbf{B}_{\mathbf{k}}$ was that the included angle $\omega^{\prime}$ between successive vectors $\mathbf{B}_{\mathbf{k}-1}$ and $\mathbf{B}_{\mathbf{k}}$ exceed $30^{\circ}$ (Barnstorf, 1980 , used $30 \mathrm{~s}$ samples). Thereafter, the additional checking interrogations were performed to optimize the proper identification of these features, so that noise, highly disturbed situations, chains of very rapid direction changes, long-lasting field gradients or longer period waves are not mistakenly accepted in the selection process. These requirements are: (1) the 
included angle between the two vectors prior to the discontinuity ( $\mathbf{B}_{\mathbf{k}-2}$ and $\left.\mathbf{B}_{\mathbf{k}-3}\right)$ and after it $\left(\mathbf{B}_{\mathbf{k}+1}\right.$ and $\mathbf{B}_{\mathbf{k}+2}$ ) should both be smaller than $15^{\circ}$ (this gives a restriction on the extent of the discontinuity, roughly $2 \mathrm{~min}$, which is much beyond a typical interval of $10 \mathrm{~s}$ and it eliminates large smooth changes); (2) the included angle between the average vectors before and after the discontinuity $\left(\left[\mathbf{B}_{\mathbf{k}-2}+\mathbf{B}_{\mathbf{k}-3}\right] / 2,\left[\mathbf{B}_{\mathbf{k}+1}+\mathbf{B}_{\mathbf{k}+2}\right] / 2\right)$ must be greater than $30^{\circ}$ (this places a limit on the variability in the neighbourhood of the discontinuity). We visually verified with plots that the identification method was working properly.

The exact time delimitation of discontinuities had to be manually performed on the high resolution data as an automated procedure would require a very high sophistication level. The next step, a visual search for a relationship between Alfvén waves and discontinuities in the high-speed stream at $0.29 \mathrm{AU}$ became much more difficult than is usual at large heliocentric distances. This happened because there is a very rich and broad fluctuations spectrum, particularly on the scale of a few minutes and even less, which has not undergone significant attenuation in the solar wind close to the Sun. Then, phase-steepened waves may be masked, or even contrarily, be feigned in this region. In view of this, we finally kept for our analysis those cases which exhibited $\omega^{\prime}>70^{\circ}$, so that the discontinuities clearly come out from the general noisy background. We had 18 initial candidates, and the verification whether each was a true phase-steepened edge of an Alfvén wave thereafter depended on a visual inspection of low resolution data with a 5 points running mean (to remove variations which obscure the identification of trends on the scale of Alfvén waves). If doubts remained after this selection method for events (slow plus fast rotation) the procedure was repeated for three point means or even taking all data into account. After this, 15 apparent events survived and a later check for high-resolution data quality left us with one less. However, further aspects to be described needed to be verified and the final number was much lower.

The measurement of the magnetic field (or any vector) in planar structures allows one to find the corresponding surface orientation through the minimum variance analysis (see e.g. Sonnerup and Cahill, 1967; Lichtenstein and Sonett, 1980). This will be applied here to study some characteristics of the observed discontinuities and Alfvén waves, and thereafter to determine if a link between each pair exists. If a fluctuation stems from a plane wave, then the directions of minimum variance and phase propagation coincide and are both normal to the constant phase surfaces. Moreover, if the waves move along the magnetic field lines, we should expect the direction to be aligned with $\mathbf{B}_{\mathrm{o}}$. In fact, it has been observed (see e.g. Klein et al., 1991) that the Alfvén wave normals are usually nearly parallel to the mean field $\left(\beta_{w} \approx 0^{\circ}\right)$. However, the orientation of rotational discontinuities $\left(\beta_{D}\right)$ may depart more significantly (see Barnstorf, 1980; Lepping and Behannon, 1986).
The minimum variance analysis gives an orientation $\hat{\mathbf{n}}$ where the IMF exhibits the least variation and it is associated with an eigenvector with the minimum eigenvalue $\lambda_{m}$. In the case of a discontinuity this direction becomes interpreted as the normal to its surface. The two further eigenvectors are associated with eigenvalues $\lambda_{I}$ and $\lambda_{M}$ of intermediate and maximum variance. If $\lambda_{I} / \lambda_{m} \gg 1$ there exists a well-defined minimum variation direction around which the perturbation vector rotates. If $\lambda_{M} / \lambda_{I} \approx 1$ or $\gg 1$ the polarization is, respectively, circular or linear, and elliptical if in between. Unreasonably large errors in the estimates of discontinuity normals occur if $\lambda_{I} \approx \lambda_{m}$, so the usual requirement $\lambda_{I} / \lambda_{m}>2$ (Sonnerup and Cahill, 1967; Lepping and Behannon, 1980) was applied. To ensure that each wave was planar it was also required that $\lambda_{I} / \lambda_{m}>2$ for the whole event (the interval of the discontinuities is negligible, being on average $3 \%$ of the total time). Notice that this $(M, I, m)$ reference system will not coincide with the one obtained for the isolated discontinuity; in particular, the angle between both surface normals could be significant.

When the discontinuity rotation angle in the maximum-intermediate plane $\omega<30^{\circ}$, the results also lose reliability (Sonnerup, 1971; Burlaga et al., 1977; Lepping and Behannon, 1980). This provides another admissibility condition. It should be noticed that the angle of the magnetic field change $\omega^{\prime}$ found from our original search for discontinuities from consecutive $40.5 \mathrm{~s}$ averages will probably never coincide with the value across the narrow current sheet $\omega^{\prime \prime}$ (given by the first and last IMF vector of the analyzed high-resolution interval) and should rather be associated with a variation between the neighbourhoods before and after. A detailed study of discontinuities must be restricted, however, to the small zone of rapid magnetic field direction change. It must be stressed that in previous works there is not always a clear distinction whether $\omega$, $\omega^{\prime}$ or $\omega^{\prime \prime}$ should be $>30^{\circ}$.

The normal field component to the discontinuity surface gives a clue whether the abrupt change is of tangential $(\approx 0)$ or rotational $(\neq 0)$ nature (see e.g. Neugebauer et al., 1984). It is basically possible to distinguish them only if combined field and plasma data are available (see Hudson, 1970), but even then ambiguities may appear. However, one may draw a distinction as to kind with high-resolution magnetic field data, as a result of the determination of $B_{n}$ with the minimum variance analysis. The borderline between both types is diffuse. This led to different separation criteria in diverse works. In order to eliminate tangential discontinuities, we withdrew the cases where $\left|\left\langle B_{n}\right\rangle\right| /\langle|\mathbf{B}|\rangle<0.3$ (Barnstorf, 1980) (i.e. the angle between $\hat{\mathbf{n}}$ and $\mathbf{B}_{\text {o }}$ $0^{\circ}<\beta_{D}<73^{\circ}$ ). It was also required that for a rotational case $\sigma_{B} / B<0.1$ (see Lepping and Behannon, 1986), where $\sigma_{B}$ is the standard deviation of the magnetic field magnitude (low and high resolution have been respectively used for the computation in waves and discontinuities). Finally five cases that complied with all constraints emerged out of the previously remaining fourteen. 
Further physical parameters were used to describe and test in detail a possible relationship between each Alfvén wave and the corresponding rotational discontinuity. The thickness of the latter $D$ is given by (Burlaga et al., 1977)

$D=V n_{r} t$

where $t$ is the corresponding observation interval, $n_{r}$ the surface normal's radial component and $V$ the solar wind speed.

The plasma polarization of waves and discontinuities $P_{W}$ and $P_{D}$ was determined from the following procedure. First one has to find out whether the wave or discontinuity moves outwards or inwards with respect to the solar wind flow by calculating $p\left(\mathbf{V}_{\mathbf{i}}-\mathbf{V}_{\mathbf{f}}\right) \cdot\left(\mathbf{B}_{\mathbf{i}}-\mathbf{B}_{\mathbf{f}}\right)$, where $p$ refers to the mean magnetic field polarity ( $p=1$ for all cases, see Fig. 1) and $\mathbf{i}, \mathbf{f}$ refer to the initial and final values. If the result is negative (positive) then the propagation is away from (towards to) the Sun. All structures were found to propagate outwards (as expected at this heliocentric distance). Then, from the two possible orientations of $\hat{\mathbf{n}}$, the one pointing into the outer half-space has to be chosen in all our cases. The plasma polarization is then found from

$$
P_{W, D}=\operatorname{sign}\left[\hat{\mathbf{n}} \cdot\left(\mathbf{B}_{\mathbf{i}} \times \mathbf{B}_{\mathbf{f}}\right)\langle\mathbf{B}\rangle \cdot \hat{\mathbf{n}}\right]
$$

where positive and negative respectively mean anti- and clockwise gyration when facing the normal. Care must be taken in using this expression because the sign must be inverted in case the angle from $\mathbf{B}_{\mathbf{i}}$ to $\mathbf{B}_{\mathbf{f}}$ exceeds $180^{\circ}$ (from the two angles between $\mathbf{B}_{\mathbf{i}}$ and $\mathbf{B}_{\mathbf{f}}$, the one running through the intermediate vectors is the appropriate one), in which case the cross product refers to the opposite rotational sense. Since in all five cases the waves and discontinuities propagate outwards and the mean IMF points in the same sense, the second dot product is always positive and $P_{W, D}$ is then equivalent here to the gyration sense of each analyzed structure. From the results for each event it is possible to determine whether it is an arc-polarized phase-steepened wave, a kind of arc-like rotation in a plane (Tsurutani et al., 1994) or if it is elliptical. It has been suggested that the former cases propagate in the intermediate variance direction (Tsurutani et al., 1997b).

The following angles were also calculated: $\omega_{W}$ of rotation of the magnetic field for the slow portion in the maximum-intermediate plane, $\psi$ between $\mathbf{B}_{\mathrm{o}}$ and the radial direction, and $\alpha$ between the wave and discontinuity normals. It should be noted that the last angle must be particularly small for an ideal event, as it is an indicator of whether its two portions have the same gyration axis. Then, it may be considered a crucial parameter in deciding if the fast and slow parts are independent of each other or form together an integral phenomenon.

\section{Examination of events}

The evolution of each case can be visualized in a $3 \mathrm{D}$ plot, showing $\mathbf{B}_{\mathbf{M}}$ and $\mathbf{B}_{\mathbf{I}}$ as a function of time (see
Figs. 2-6), where we have used the field minimum variance system determined from the analysis of the whole wave plus discontinuity interval (like Tsurutani et al., 1997a). In Table 1 we present various characteristics and calculated parameters, which provide additional tools for the description of each event. The angles of discontinuities $\omega$ may not totally mimic those in the figures, as the curves were constructed with $2 \mathrm{~s}$ (events 3 and 5) or $10 \mathrm{~s}$ (events 1,2 and 4) averages to avoid large dark surfaces, which would obscure the apparent observation in three dimensions. Although arc-like or elliptical cases may be visually recognized, due to the perspective this may not always be completely clear. Then, the joint knowledge of the plasma polarization state of both wave and discontinuity in each event might be more appropriate for a correct determination. We now analyze the results in each case.

\section{Event 1}

This is the only one of the five cases where the discontinuity has been found at the leading edge. Therefore Fig. 2 is presented as seen from the back. The field rapidly completes almost half of a full turn in $28 \mathrm{~s}$ (discontinuity), but then slowly rotates back in 22 min (Alfvén wave), which as a whole comprises a kind of $360^{\circ}$ rotation in a $2 \mathrm{D}$ plane, an arc-polarized phase-steepened wave. As this kind of wave is supposed to propagate in the intermediate direction, the angle between the wave and discontinuity intermediate axes was checked. It gave $25^{\circ}$, and therefore provided no particular clue, as this is the same value as $\alpha$. The other parameters in Table 1 are in good agreement with expected values for an event.

\section{Event 2}

In Fig. 3, an anticlockwise gyration of the tip takes $14 \mathrm{~min}$ to complete almost $180^{\circ}$, and then in only $48 \mathrm{~s}$ it returns in the opposite sense by a similar amount, again describing a kind of arc in the maximum-intermediate

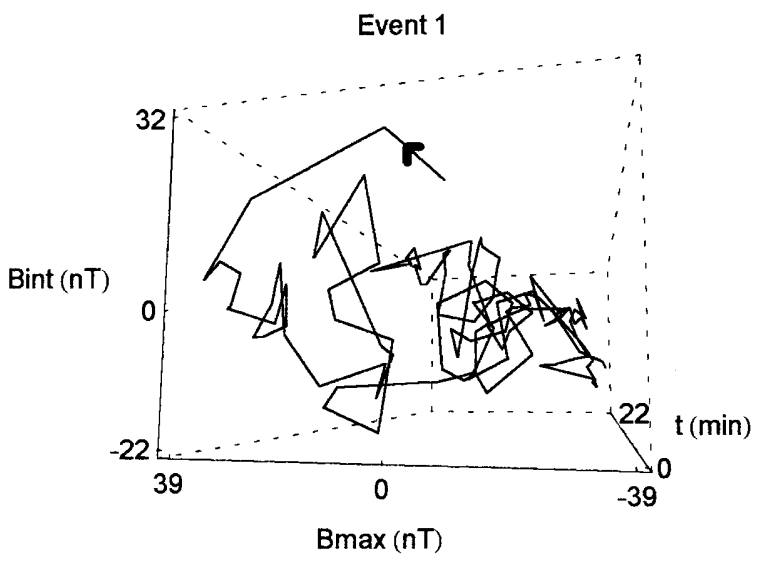

Fig. 2. The evolution in time of event 1 
Table 1. Characteristics and parameters of the events

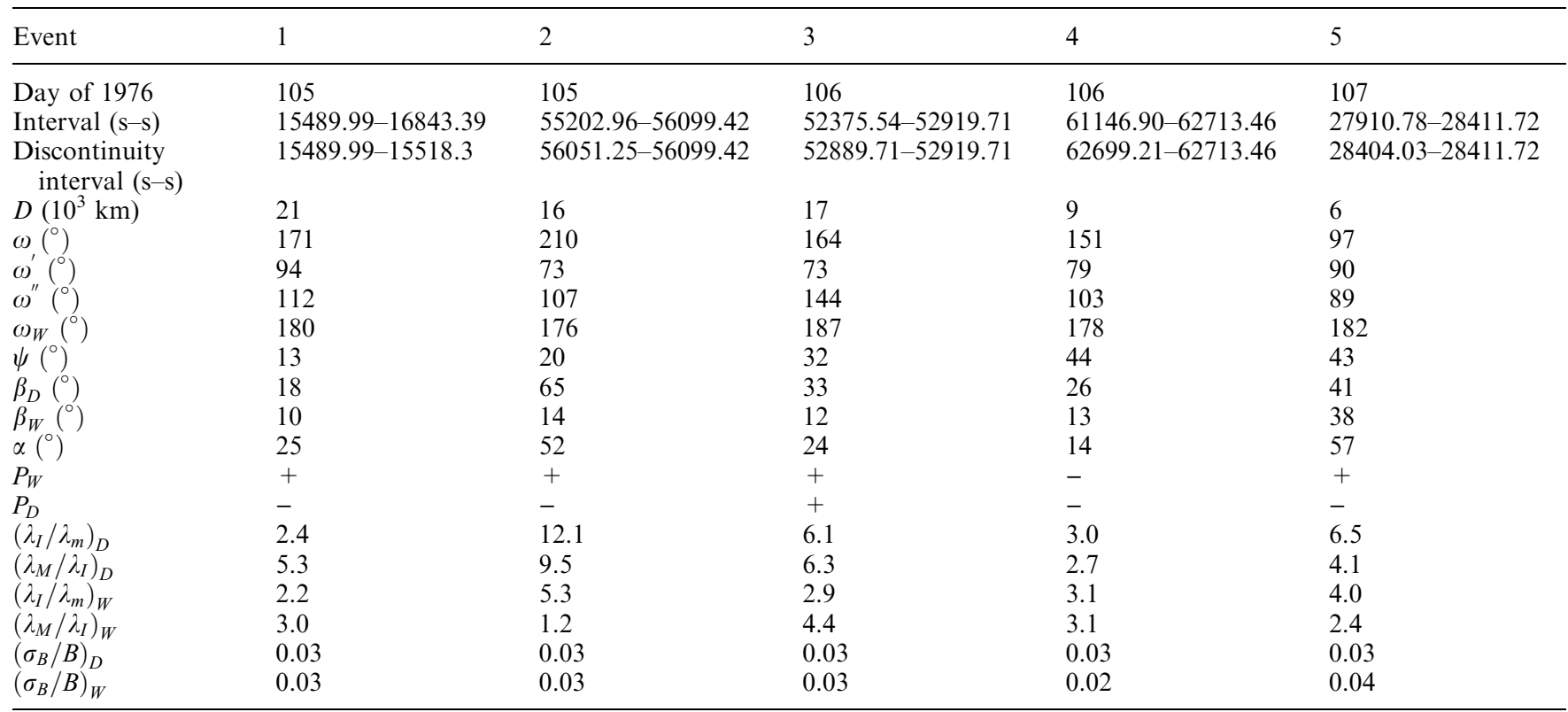

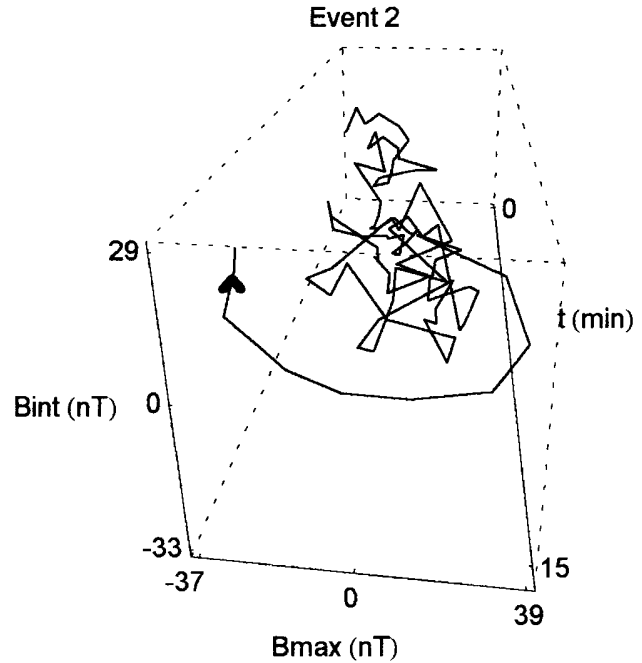

Fig. 3. The evolution in time of event 2

plane. From the visual point of view this case seems to be nearly ideal. However, $\beta_{D}$ and $\alpha$ both exhibit large values. The calculation of the angle between the intermediate direction of the wave and the discontinuity normal gives $56^{\circ}$, so this does not remove the problem that $\alpha$ is too large. Then, it appears that this case only had resembled of a phase-steepened Alfvén wave, but had to be rejected as that. It is also worth noting that there is a very large variability among the values of the $\lambda$ ratios for this event in Table 1.

\section{Event 3}

The field rotates in Fig. 4 about $180^{\circ}$ in 9 min and then almost completes the $360^{\circ}$ in $30 \mathrm{~s}$, drawing a kind of ellipse in the $2 \mathrm{D}$ plane. The parameters $\beta_{D}, \beta_{W}, \alpha$ in Table 1 all look satisfactory.

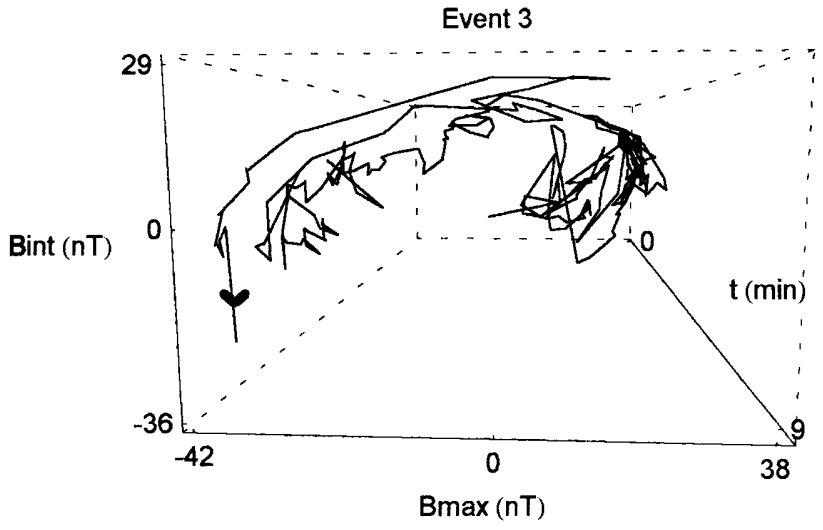

Fig. 4. The evolution in time of event 3

\section{Event 4}

The evolution of this case shows a rotation about $180^{\circ}$ in almost half an hour and then a near completion of an ellipse in $14 \mathrm{~s}$. The parameters $\beta_{D}, \beta_{W}$ and $\alpha$ in Table 1 are all very reasonable. However, it is curious that the average IMF exhibits here a notorious departure from the expected radial direction, but this does not prohibit the occurrence of the phenomenon.

\section{Event 5}

This is a doubtful case. Although it fulfilled the previous requirements of our selection process it finally exhibits half a turn in $8 \mathrm{~min}$ and a further quarter in $8 \mathrm{~s}$ and therefore does not make up one full rotation. The visual inspection of Fig. 6 does not show an example as clear as previous ones. Moreover, $\beta_{D}, \beta_{W}$ and $\alpha$ are all very large, contrary to the optimum null value. The mean 


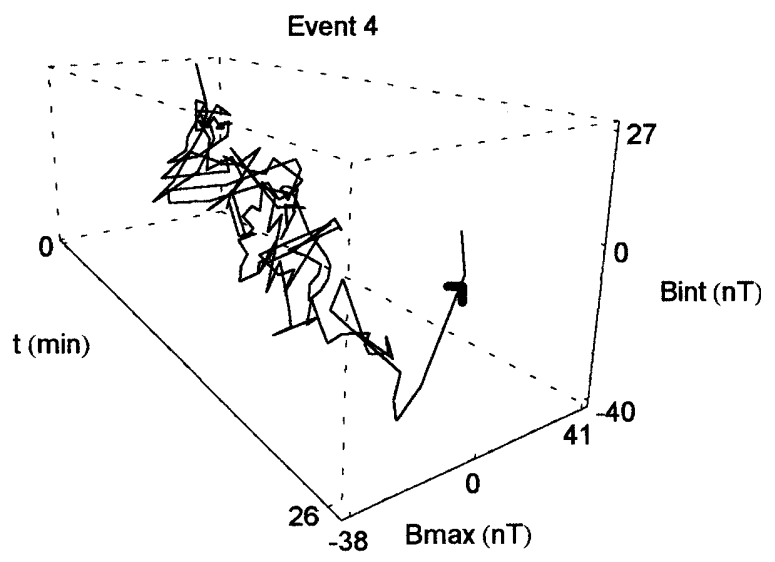

Fig. 5. The evolution in time of event 4

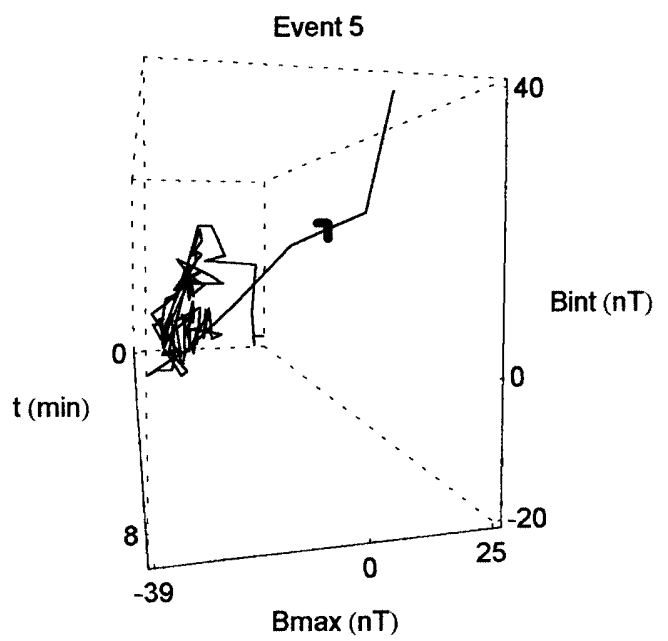

Fig. 6. The evolution in time of event 5

total field also presents a remarkable separation from the radial orientation.

It may be seen that the calculation of $\beta_{D}, \beta_{W}$ and $\alpha$ is a useful procedure in the verification of true events, and the finding of $P_{W, D}$ of discontinuities and waves is appropriate for the determination of the polarization of the whole phase-steepened waves. Only three cases $(1,3$ and 4) out of five survived the scrutiny of our additional parameters. In case it is not just a simple coincidence, no explanation could be found for the disappearance of events during the last three days of our interval, although the Alfvénic correlations were still high. The prevalence of left-hand rotations in the discontinuities cannot be considered statistically significant due to the small number of cases studied. In fact, this dominance should be expected (Barnstorf, 1980) only if the typical thickness of the discontinuities $\left(\sim 10^{4} \mathrm{~km}\right.$ in our events) was smaller than a few proton gyroradii $(\sim 30 \mathrm{~km}$ at $0.3 \mathrm{AU})$.

\section{Conclusions}

The data of the present analysis belongs to a fast stream found in one of the closest approaches by a spacecraft to the Sun. Ideal Alfvénic conditions would be expected for this observation interval and were in fact verified. This is an optimum scenario for the occurrence of phase-steepened Alfvén waves.

A careful search process of these events, similar to previous work, was applied to our database. The calculation of some additional parameters within the cases that emerged has been suggested and tested here for a proper determination of phase-steepened waves. For example, one of the events seemed to be nearly ideal according to a graphical representation and also due to the study of the behaviour of the physical magnitudes used in those earlier analyses. However, it had to be discarded as a true case after the constraints of our further diagnostic implements were imposed. It is suggested that these new requirements be taken into account in future identifications of cases.

The calculation of the polarization state of both wave and discontinuity in each event might be more appropriate than a visual inspection of $3 \mathrm{D}$ plots for a correct determination of the polarization of the overall waves, which were either arc-like or elliptical in our cases. The accompanying discontinuities have been found at either the leading or the trailing edges of the waves.

Contrary to the expectations due to the a priori ideal conditions for the occurrence of the events, only three good cases out of five days were found. It is believed that the search became difficult due to the rich short-scale spectrum present. Then, optimum conditions for the detection of these events may have to be restated as they might be different than those just for their existence.

Acknowledgements. The idea for this work was suggested by Prof. E. Marsch during my stay at the Max Planck Institut in Lindau on September 1997. The principal investigators respectively for the Helios plasma and magnetometer instruments are Dr. H. Rosenbauer and Prof. F. Neubauer. The production of the $40.5 \mathrm{~s}$ Helios data set was by Dr. R. Schwenn and Dr. K. Ivory. I wish to thank Drs. B. Tsurutani and A. Viñas for helpful comments and Drs. K. Ivory and A. Söding for making the lower and high-resolution data transfer and use possible. I also would like to express my gratitude to two unknown referees for helpful suggestions. P.A. is a member of Conicet.

Topical Editor E. Antonucci thanks R. P. Lepping and another referee for their help in evaluating this paper.

\section{References}

Barnstorf, H., Stromschichten im interplanetaren Plasma, PhD Thesis, Technische Universität Braunschweig, Germany, 1980.

Belcher, J. W., and L. Davis, Large-amplitude Alfvén waves in the interplanetary medium, 2, J. Geophys. Res., 76, 3534, 1971.

Burlaga, L. F., Micro-scale structures in the interplanetary medium, Solar Phys., 4, 67, 1968.

Burlaga, L. F., Directional discontinuities in the interplanetary magnetic field, Solar Phys., 7, 54, 1969.

Burlaga, L. F., Interplanetary magnetohydrodynamics, Oxford University Press, New York, 1995.

Burlaga, L. F., J. F. Lemaire, and J. M. Turner, Interplanetary current sheets at 1 AU, J. Geophys. Res., 82, 3191, 1977.

Coleman, P. J., Turbulence, viscosity and dissipation, in the solar wind plasma, Astrophys. J., 153, 371, 1968.

Hudson, P. D., Discontinuities in an anisotropic plasma and their identification in the solar wind, Planet. Space Sci., 18, 1611, 1970. 
Klein, L. W., D. A. Roberts, and M. L. Goldstein, Anisotropy and minimum variance directions of solar wind fluctuations in the outer heliosphere, J. Geophys. Res., 96, 3779, 1991.

Lepping, R. P., and K. W. Behannon, Magnetic field directional discontinuities: 1, minimum variance errors, J. Geophys. Res., $\mathbf{8 5}, 4695,1980$.

Lepping, R. P., and K. W. Behannon, Magnetic field directional discontinuities: characteristics between 0.46 and $1.0 \mathrm{AU}$, J. Geophys. Res., 91, 8725, 1986.

Lichtenstein, B. R., and C. P. Sonett, Dynamic magnetic structure of large amplitude Alfvénic variations in the solar wind, Geophys. Res. Lett., 7, 189, 1980.

Marsch, E., MHD turbulence in the solar wind, in Physics of the inner heliosphere II, Eds. R. Schwenn and E. Marsch, Springer, Berlin Heidelberg New York, 1991.

Neugebauer, M., D. R. Clay, B. E. Goldstein, B. T. Tsurutani, and R. D. Zwickl, A reexamination of rotational and tangential discontinuities in the solar wind, J. Geophys. Res., 89, 5395, 1984.

Sonnerup, B. U. O., Magnetopause structure during the magnetic storm of September 24, 1961, J. Geophys. Res., 76, 6717, 1971.

Sonnerup, B. U. O., and L. J. Cahill, Magnetopause structure and attitude from Explorer 12 observations, J. Geophys. Res., 72, $171,1967$.
Tsurutani, B. T., and E. J. Smith, Interplanetary discontinuities: temporal variations and the radial gradient from 1 to $8.5 \mathrm{AU}$, J. Geophys. Res., 84, 2773, 1979.

Tsurutani, B. T., C. M. Ho, E. J. Smith, M. Neugebauer, B. E. Goldstein, J. S. Mok, J. K. Arballo, A. Balogh, D. J. Southwood, and W. C. Feldman, The relationship between interplanetary discontinuities and Alfvén waves: Ulysses observations, Geophys. Res. Lett., 21, 2267, 1994.

Tsurutani, B. T., K. H. Glassmeier, and F. M. Neubauer, A review of nonlinear low frequency (LF) wave observations in space plasmas: on the development of plasma turbulence, in Nonlinear waves and chaos in space plasmas, ch. 1, Terrapub, Tokyo, 1997a.

Tsurutani, B. T., C. M. Ho, J. K. Arballo, G. S. Lakhina, K. H. Glassmeier, and F. M. Neubauer, Nonlinear electromagnetic waves and spherical arc-polarized waves in space plasmas, Plasma Phys. Control Fusion, 39, 237, 1997b.

Tu, C. Y., and E. Marsch, The evolution of MHD turbulence in the solar wind, Eds. E. Marsch and R. Schwenn, Solar Wind Seven, Cospar Colloquia Series, 3, Pergamon Press, Oxford, 549, 1992.

Tu, C. Y., and E. Marsch, MHD structures, waves and turbulence in the solar wind: observations and theories, Space Sci. Rev., 73, 1, 1995.

Turner, M. T., On the relation between solar wind structure and wind rotational and tangential discontinuities, J. Geophys. Res., 78, 59, 1973. 